\title{
Nasal Valve Surgery: How I Do It?
}

\author{
Abdul Rahman Al Ghareeb, Jitendra Nagarbhai Patel, Mostafa Bakry \\ Ear, Nose \& Throat Sleep Well Clinic, Noor Specialist Hospital, Manama, Bahrain \\ Email: entsleepwellclinic@hotmail.com
}

Received November 27, 2012; received December 25, 2012; accepted January 5, 2013

\begin{abstract}
One of the reasons for patients attending ENT clinic with a persistent feeling of Nasal obstruction is the presence of a narrow nasal valve. Currently, there are many surgical methods for widening narrow nasal valves. Yet, most of these methods are difficult to perform and with unpredictable results. The main purpose of this study is to describe and evaluate our technique of minimally invasive nasal valve surgery. Thirty three patients, who attended our clinic complaining from of nasal obstruction, were evaluated for indication for nasal valve surgery, complications, and postoperative results. Thirty one out of the 33 patients (94\%) who underwent the surgery reported subjective improvement. Visual analogue score (VAS score) was used to estimate the degree of pre and postoperative nasal obstruction, the mean preoperative score was 8.891 and the mean postoperative was 3.241 and the improvement was statistically significant $(\mathrm{P}<$ 0.002), ( $\mathrm{R}=3.174)$. In conclusion, it can be said that the technique of Minimal Invasive Nasal Valve Surgery was found to be safe and extremely effective for most patients having narrow nasal valve.
\end{abstract}

Keywords: Nasal Obstruction; Nasal Valve Narrowing; Nasal Valve Surgery

\section{Introduction}

The nasal valve area is not a singular structure, but a complex three-dimensional construct consisting of several morphological structures. From the physiologic point of view, it is the place of maximum nasal flow resistance ("flow limiting segment") [1].

The nasal valve is subdivided into internal and external components. The internal nasal valve is formed by the articulation of the upper lateral cartilages with the cartilaginous septum. The angle of articulation at this site is normally $10^{\circ}$ to $15^{\circ}$. The external nasal valve is bounded superolaterally by the caudal edge of the upper lateral cartilages. The lateral border is the bony pyriform aperture of the maxilla and fibrofatty tissue of the ala. Support for this lateral border area is provided by the ligamentous attachment of the lateral crus to the bony maxilla. Inferiorly, the external valve is limited by the nasal floor and posteriorly by the head of the inferior turbinate [2].

Concerning the nasal valve, there are only two important muscles with opening function. The first one is the M. dilatator naris that originates at the lateral crus of the alar cartilage and inserts into the skin of the wing of the nose. It has a stabilizing function on the external nasal valve and an indirect effect on the nasal valve through a consecutively caused outward deflection of the caudal end of the lateral cartilage and thus opening of the area of the nasal valve. The second important muscle is the pars alaris of the M. nasalis. It originates in the incisive fossa of the maxilla and inserts at the accessory cartilages and the skin in the region of the hinge area. This enables it to pull this structure in the lateral direction and widen the internal nasal valve $[1,3]$.

Aim of this study is to show that patients presenting with persistent nasal obstruction should always be evaluated for the possibility of a narrow nasal valve and such nasal valve can be effectively treated by minimal invasive surgery.

\section{Classification, Diagnosis and Treatment of Nasal Valve Disorders}

We have to distinguish between static and dynamic disorders of the nasal valve. However, both disorders can occur at the same time and/or be interdependent.

Causes of static disorders include hypertrophy of the head of the lower turbinate, nasal septum deviations, bony constrictions of the piriform aperture, anatomic variations of the cartilaginous lateral nasal wall or scarred stenoses of the nasal valves. Furthermore, neurogenic causes (facial nerve paralysis, stroke) can result in a symptomatic impairment of nasal breathing through distortion of the lateral nasal wall.

Dynamic causes includes weakness in the alar cartilages which leads to their inversion during inspiration $[4,5]$. The most common causes of nasal valve obstruction are previous rhinoplasty (79\%), followed by nasal 
trauma (15\%) and congenital anomaly (6\%) [6].

Nasal valve disorders are frequently overlooked and/or not covered in a systematic examination. The patient's history is very important in the investigation of impairments of nasal breathing. It provides an indication of the presence of pathologies in the region of the nasal valve area and in particular of the subjective estimation of the severity of the symptoms [1].

In addition, maneuvers performed during clinical examination can diagnose a nasal valve disorder. One of the recommended maneuvers is Cottle's maneuver. It was described for the first time by Heinberg and Kern in 1973. It involves widening of the nasal valve area by pulling it in lateral direction in the area of the nasolabial groove. An improvement of nasal breathing indicates an involvement of the valve. Currently, flexible nasal endoscopy is the standard tool for the exploration and assessment of the nasal valve area.

Treatment of nasal valve disorders includes non-surgical and surgical therapies. Non-surgical therapies involves widening of the nasal valve area has been carried out for a long time using self-holding dilators (made out of wire, rubber, celluloid or other materials) that are worn when not in public (in most cases at night). Various surgical techniques have been described in the literature to address the nasal valve, including alar batten grafts, spreader grafts, flaring sutures, overlay grafts, and lateral suture suspensions [1].

\section{Material and Methods}

Thirty three patients attended ENT Sleep Well Clinic complaining from persisting symptoms of nasal obstructtion; almost all of them had treatment before attending the clinic either surgical or medical with intranasal steroids, decongestants, antihistamines and intranasal saline irrigation, both of which had minimal effect to relive their symptoms. The patients were evaluated and the diagnosis of nasal valve insufficiency was confirmed using both anterior rhinoscopy and nasal endoscopy. Cottle's maneuver was performed and recorded if it relieved the nasal obstruction.

Operating method: All of the patients underwent the technique of Minimal Invasive Nasal Valve Surgery to correct the nasal valve collapse. The procedure is performed with under local anesthesia which is obtained by injection of lidocaine $1 \%$ with 1:100,000 epinephrine into the nasal valve region. Nasal cavity is then prepared with betadine solution. An intercartilaginous incision is then performed on the area of the caudal part of the upper lateral cartilage. Elevation of the mucopericondrial flap, the caudal border of the upper lateral cartilage is then identified and removal of $5 \mathrm{~mm}$ piece of cartilage is accomplished. Suturing of the incision is done using two 4-0 absorbable vycril sutures (Figure 1). Antibiotic oint-

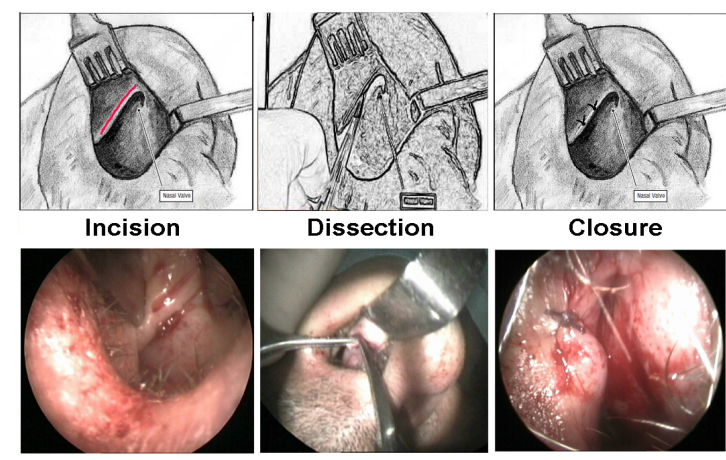

Figure 1. The steps of Nasal Valve Minimal Invasive Procedure: the incision, dissecting the inferior border of upper lateral cartilage and stitching at the end of the procedure.

ment is then applied to the vestibule of the nose. The patient is given oral antibiotic with oral pain medications. The operation is video recorded using LEMO FGG.2B Camera and DVD recorder after which still photo is then captured. Visual analogue score (VAS) was used to estimate the degree of preoperative and postoperative nasal obstruction with asking the patient to detect the degree of their nasal obstruction on a scale of 0 to 10 with 10 being total nasal obstruction and 0 being perfect nasal airflow [21], also the patients were asked for subjective improvement.

\section{Results}

From the thirty three patients who had attended the ENT Sleep Well Clinic complaining from symptoms of nasal obstruction, 5 patients had undergone previous nasal surgeries like septoplasty, turbinectomy, Fess and Rhinoplasty. Out of the 5 patients who underwent surgeries, 3 had slight adhesions and all had persistent symptoms post operatively.

A Total of 33 patients, 27 (81.8\%) males and 6 (18.2\%) females underwent the nasal valve surgery. The procedure was bilateral in 28 patients and unilateral in 5 patients. The mean age of the patients was 43 years with a range of 22 - 64 years.

Thirty one out of the 33 patients (94\%) who underwent the surgery reported subjective improvement, while only 2 patients reported no such improvement (Figure 2). Visual analogue score (VAS) was used to estimate the degree of nasal obstruction preoperatively and 3 months postoperatively. On a scale of 0 - 10, with 10 indicating total nasal obstruction and zero no obstruction, the mean preoperative score was 8.891 and the mean postoperative was 3.241. Statistical analysis using paired $t$ test and correlation coefficient showed that the improvement in the postoperative nasal obstruction score was statistically significant $(\mathrm{P}<0.002)$, $(\mathrm{R}=3.174)$.

Complications were unremarkable with one patient suffered from increased postoperative snoring loudness 


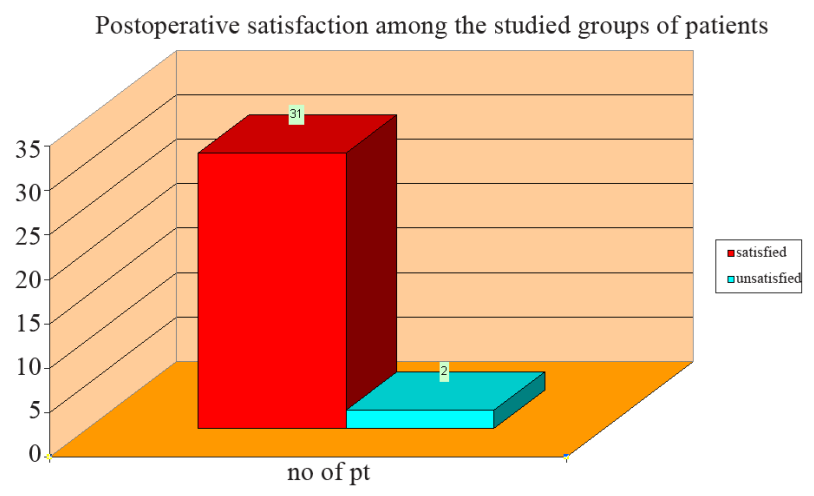

Figure 2. Postoperative degree of satisfaction among the studied patients.

and two patients from recurrence of the original symptoms.

\section{Discussion}

Many procedures have been developed to tackle the problem of nasal valve insufficiency. Spreader grafts was used between upper lateral cartilage and the septum [15], flaring sutures [20], butterfly grafts [22] and orbital suspension procedures [18]. Other procedures used to repair the internal nasal collapse involved removal of a part of the caudal portion of upper lateral cartilage like Z-plasty [21], intranasal M-plasty [23]. These are similar to our procedure in the location of the primary incision and the removal of a strut of the caudal boarder of the upper lateral cartilage.

The Z-plasty uses triangular flaps to further alter and lateralize the scroll region. The removal of the caudal upper lateral cartilage is somewhat counterintuitive with both procedures but is necessary to normalize the valve angles and in the case of the Z-plasty to mobilize the flaps. Flap mobilization also creates mild cephalic rotation of the nostrils, which further improves nasal airflow, and that removal of a cephalic strip of alar cartilage in the scroll region, or in this case removal of the adjacent caudal border of the upper lateral cartilage, creates a cartilaginous void in the scroll region and allows for rotation of the tip, although this is not severe enough to cause significant cosmetic deformity [21].

Advantage of our technique is that it is a simple technique to repair the collapsed nasal valve when compared to the others since it widens the nasal valve angle and makes unremarkable tip rotation of which both can lead to improve of nasal obstruction (Figure 1).

In the suspension technique reported by Andre \& Vuyk [24], five patients (25\%) had post operative complications: one patient experienced temporary tenderness between the orbital rim and the nose, one had relatively mild complaints of a slight thickness under the eye and three experienced a painful swelling under the eye, which did not respond to antibiotic treatment. On the contrary, in our serious there were unremarkable complications in three patients: two patients complained from recurrence of nasal obstruction and one patient suffered from increased degree of snoring. So, it is another advantage of our technique that post-operative very less incidence of complications.

The study showed a statistically significant subjective improvement in the nasal valve obstruction based on a mean preoperative VAS score of 8.891 and a postoperative score of $3.241(\mathrm{P}<0.002)$, and a nasal endoscopy viewed by the operating surgeon which revealed a subjective widening of the valve angle (Figure 2). Subjectively, 31 patients were satisfied and 2 unsatisfied. This matches with the results from other studies using nearly same technique $[21,25]$.

Our intranasal technique offers several advantages over the other techniques used to repair internal nasal valve. First, it is done under local anesthesia and can be performed in the outpatient clinic. Second, it is performed intranasaly with minimal dissection and doesn't require tissue grafting. Third, suturing the incision leads to proper healing and direct the fibrosis laterally leading to further widening of the valve. Finally, it has an unremarkable postoperative rate of complications.

\section{Conclusion}

Patients presenting with persistent nasal obstruction should always be evaluated for the possibility of a narrow nasal valve. In our experience, the technique of Minimal Invasive Nasal Valve Surgery was found to be safe and extremely effective for most patients and we do recommend it as a first line surgical treatment for internal nasal valve collapse. Further objective studies for evaluation of widening of nasal valve using acoustic rhinomanometery is recommended.

\section{REFERENCES}

[1] Marc Boris Bloching, "Disorders of the Nasal Valve Area," Otolaryngology: Head and Neck Surgery, Vol. 6, 2007, p. Doc07.

[2] M. Khosh, J. Albert, H. Carlo and P. Steven, "Nasal Valve Reconstruction," Archives of Facial Plastic Surgery, Vol. 6, No. 3, 2004, pp. 167-171. doi:10.1001/archfaci.6.3.167

[3] D. B. Wexler and T. M. Davidson, "The Nasal Valve: A Review of the Anatomy, Imaging, and Physiology," The American Journal of Rhinology, Vol. 18, No. 3, 2004, pp. 143-150.

[4] T. Bruintjes, A. Olphen, B. Hillen and E. A. Huizing, "Functional Anatomic Study of the Relationship of the Nasal Cartilages and Muscles to the Nasal Valve Area," Laryngoscope, Vol. 108, No. 7, 1998, pp. 1025-1032. doi:10.1097/00005537-199807000-00014 
[5] M. Vaiman, E. Eviatar and S. Segal, "Intranasal Electromyography in Evaluation of the Nasal Valve," Rhinology, Vol. 41, No. 3, 2003, pp. 134-141.

[6] M. Constantian, "Differing Characteristics in 100 Consecutive Secondary Rhinoplasty Patients Following Closed versus Open Surgical Approaches," Plastic and Reconstructive Surgery, Vol. 109, No. 6, 2002, pp. 2097-2111. doi:10.1097/00006534-200205000-00048

[7] E. Ricci, F. Palonta, G. Preti, N. Vione, G. Nazionale, R. Albera, A. Staffieri, G. Cortesina and A. L. Cavalot, "Role of Nasal Valve in the Surgically Corrected Nasal Respiratory Obstruction: Evaluation through Rhinomanometry," American Journal of Rhinology, Vol. 15, No. 5, 2001, pp. 307-310.

[8] R. Gruber, A. Lin and T. Richard, "Nasal Strips for Evaluating and Classifying Valvular Nasal Obstruction,” Aesthetic Plastic Surgery, Vol. 35, No. 2, 2011, pp. 211-215. doi:10.1007/s00266-010-9589-4

[9] T. Keck, R. Leiacker, S. Kühnemann, J. Lindemann, A. Rozsasi and N. Wantia, "Video-Endoscopy and Digital Image Analysis of the Nasal Valve Area," European Archives of Oto-Rhino-Laryngology, Vol. 263, No. 7, 2006, pp. 675-679. doi:10.1007/s00405-006-0025-y

[10] O. Hillberg, A. C. Jackson, D. L. Swift and O. F. Pederson, "Acoustic Rhinometry: Evaluation of Nasal Cavity Geometry by Acoustic Reflections,” Journal of Applied Physiology, Vol. 66, No. 1, 1989, pp. 295-303.

[11] B. K. Howard and R. J. Rohrich, "Understanding the Nasal Airway: Principles and Practice,” Plastic and Reconstructive Surgery, Vol. 109, No. 3, 2002, pp. 1128-1144. doi:10.1097/00006534-200203000-00054

[12] F. Noltenius, "Ein Einfaches Verfahren zur Symptomatischen Linderung der Ozaenabeschwerden," European Archives of Oto-Rhino-Laryngology, Vol. 130, No. 4, 1932, p. 358. doi:10.1007/BF01591456

[13] T. C. Amis, J. P. Kirkness, E. Di Somma and J. R. Wheatley, "Nasal Vestibule Wall Elasticity: Interactions with a Nasal Dilator Strip," Journal of Applied Physiology, Vol. 86, No. 5, 1999, pp. 1638-1643.

[14] B. Guyuron, B. Michelow and C. Englebardt, "Upper Lat Eral Splay Graft," Plastic and Reconstructive Surgery, Vol. 102, No. 6, 1998, pp. 2169-2177. doi:10.1097/00006534-199811000-00058

[15] R. F. André, S. H. Paun, H. D. Vuyk, “Endonasal Spreader Graft Placement as Treatment for Internal Nasal Valve
Insufficiency: No Need to Divide the Upper Lateral Cartilages from the Septum," Archives of Facial Plastic Surgery, Vol. 6, No. 1, 2004, pp. 36-40. doi:10.1001/archfaci.6.1.36

[16] T. Akcam, O. Friedman and T. A. Cook, "The Effect on Snoring of Structural Nasal Valve Dilatation with a Butterfly Graft," Archives of Otolaryngology—Head \& Neck Surgery, Vol. 130, No. 11, 2004, pp. 1313-1318. doi:10.1001/archotol.130.11.1313

[17] S. S. Rizvi and M. G. Gauthier, "How I Do It: Lateralizing the Collapsed Nasal Valve," Laryngoscope, Vol. 113, No. 11, 2003, pp. 2052-2054. doi:10.1097/00005537-200311000-00037

[18] R. C. Paniello, "Nasal Valve Suspension,” Archives of Otolaryngology, Head and Neck Surgery, Vol. 122, No. 12, 1996, pp. 1342-1346. doi:10.1001/archotol.1996.01890240050011

[19] D. Lee and A. Galasgold, "Correction of Nasal Valve Stenosis with Lateral Suture Suspension," Archives of Facial Plastic Surgery, Vol. 3, No. 4, 2001, pp. 237-240. doi:10.1001/archfaci.3.4.237

[20] S. S. Park, "The Flaring Suture to Augment the Repair of the Dysfunctional Nasal Valve," Plastic and Reconstructive Surgery, Vol. 101, No. 4, 1998, pp. 1120-1122. doi:10.1097/00006534-199804040-00036

[21] J. Dutton and M. Neidich, "Intranasal Z-Plasty for Internal Nasal Valve Collapse,” Archives of Facial Plastic Surgery, Vol. 10, No. 3, 2008, pp. 164-168. doi:10.1001/archfaci.10.3.164

[22] J. M. Clark and T. A. Cook, “The 'Butterfly' Graft in Functional Secondary Rhinoplasty,” Laryngoscope, Vol. 112, No. 11, 2002, pp. 1917-1925. doi:10.1097/00005537-200211000-00002

[23] D. L. Schulte, D. A. Sherris and E. B. Kern, "M-Plasty Correction of Nasal Valve Obstruction,” Facial Plastic Surgery Clinics of North America, Vol. 7, No. 3, 1999, pp. 405-409.

[24] R. André and D. Vuyk, "Nasal Valve Surgery; Our Experience with the Valve Suspension Technique,” Rhinology, Vol. 46, No. 1, 2008, pp. 66-69.

[25] D. M. Toriumi, J. Josen, M. Weinberger and M. E. Tardy, "Use of Alar Batten Grafts for Correction of Nasal Valve Collapse," Archives of Otolaryngology-Head \& Neck Surgery, Vol. 123, No. 8, 1997, pp. 802-808. doi:10.1001/archotol.1997.01900080034002 